\title{
Laboreal
}

Volume $8 \mathrm{~N}^{\circ} 2$ | 2012

A antropotecnologia, ferramenta ou engodo?

\section{Antropología cognitiva y antropotecnología}

Antropologia cognitiva e antropotecnologia

Anthropologie cognitive et anthropotechnologie

Cognitive anthropology and anthropotechnology

\section{Jacques Theureau}

\section{OpenEdition}

\section{Journals}

Edición electrónica

URL: http://journals.openedition.org/laboreal/6606

DOI: 10.4000/laboreal.6606

ISSN: 1646-5237

\section{Editor}

Universidade do Porto

\section{Referencia electrónica}

Jacques Theureau, «Antropología cognitiva y antropotecnología », Laboreal [En línea], Volume 8 N² I 2012, Publicado el 01 diciembre 2012, consultado el 10 octubre 2019. URL : http:// journals.openedition.org/laboreal/6606; DOI : 10.4000/laboreal.6606

Este documento fue generado automáticamente el 10 octubre 2019.

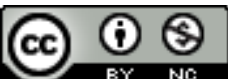

Laboreal está licenciado com uma Licença Creative Commons - Atribuição-NãoComercial 4.0 Internacional. 


\title{
Antropología cognitiva y antropotecnología
}

\author{
Antropologia cognitiva e antropotecnologia \\ Anthropologie cognitive et anthropotechnologie \\ Cognitive anthropology and anthropotechnology
}

Jacques Theureau

\section{NOTA DEL EDITOR}

Manuscrito recibido en: Enero/2012

Aceptado tras peritaje: Agosto/2012

\section{Introducción}

1 Las cuestiones abordadas en este dossier me remiten a las conversaciones con A. Wisner cuando éste inició, a finales de los años 80 del siglo pasado, a través de conferencias y de las tesis sobre ergonomía que dirigía, lo que él denominó antropotecnología. Fue en una etapa de mi trabajo de investigación en colaboración con L. Pinsky durante la cual, a raíz de unas conversaciones con L. Suchman, C. Goodwin, A. Cicourel, E. Hutchins, M. Cole y S. Scribner (en diversas ocasiones entre 1985 y 1988) y de lecturas extensivas sobre antropología cognitiva norteamericana y sobre antropología cultural, llegué a la conclusión de que la teoría y la metodología de análisis de la actividad humana que estábamos desarrollando en el marco de la investigación ergonómica (sistematizada por primera vez en Pinsky \& Theureau, 1987) estaban más próximas de lo que parecía de una "antropología cognitiva" que de las que se presentaban como "psicología cognitiva" y a las que estas se habían asimilado hasta entonces con más o menos fortuna (véase Pinsky et al., 1989, Pinsky, 1990, Theureau, 1990). Incluso me pareció que éstas podían contribuir, gracias a la relación orgánica de las investigaciones empíricas realizadas con el concepto técnico-organizacional de las situaciones de trabajo, a 
enriquecer las hipótesis teóricas y a desarrollar su carácter científico. Así, era altamente significativo para mí que A. Ombredane, cuya obra con J.-M. Faverge (Ombredane \& Faverge, 1956), fue el origen de la especificidad de la ergonomía francesa, hubiera seguido las enseñanzas de antropología teórica de M. Jousse, se hubiera interesado particularmente en las relaciones entre la palabra y la acción y hubiera publicado, entre otros, un artículo de antropología colonial sobre la construcción de casas en el ex-Congo Belga. Hice llegar a A. Wisner las publicaciones antropológicas cognitivas que nos inspiraban. B. Pavard, que en esta época se identificaba más con la "ingeniería cognitiva", también encontraba en estas publicaciones una fuente de inspiración y alimentaba asimismo a A. Wisner. De este modo podíamos conversar con todos los elementos en mano.

2 A. Wisner concebía la antropotecnología como una ergonomía de la transferencia de tecnología de los países industrializados hacia el tercer mundo que añadiría la antropología física, cognitiva y cultural a las disciplinas empíricas utilizadas tradicionalmente en las investigaciones ergonómicas, la fisiología, la psicología y la sociología. Por una parte, estimé más juicioso integrar la transferencia de tecnología en la ergonomía y contribuir así a hacer de ella una ingeniería situacional en el más amplio sentido, por otra parte, integrar las investigaciones empíricas sobre antropología física, cognitiva y cultural en la interdisciplinariedad ergonómica en general, otorgando un lugar central a una antropología cognitiva desarrollada según el paradigma cognitivo de la enacción a la que era tan aficionado y contribuir así a reforzar las relaciones orgánicas de la tecnología ergonómica con las ciencias humanas y sociales. En la actualidad, es evidente que A. Wisner tenía razón en lo referente a la consideración de la coyuntura social, económica, científica, técnica y cultural en la reflexión estratégica, científica e institucional. La antropotecnología se desarrolló, incluso en ausencia de A. Wisner, y las aportaciones de la antropología física, cognitiva y cultural a la ergonomía siguieron siendo escasas, mientras que la antropología cognitiva y la ingeniería situacional que yo desarrollaba junto con otros en el programa de investigación denominado 'curso de acción' si estas se han mantenido en el ámbito del análisis del trabajo y del concepto ergonómico (ampliado de la concepción de los espacios y de las máquinas a la de la formación, de la organización, de la gestión y de la logística), se desarrollaron más ampliamente migrando hacia otros campos, el análisis de las actividades de rendimiento, de formación y de entrenamientos deportivos y de actividades educativas, tanto en la vertiente docente como en la vertiente del alumno y su articulación, y la concepción de los entornos, herramientas informáticas y procedimientos de formación y de entrenamiento, por no hablar de mi incursión reciente en el análisis de las prácticas musicales (composición, interpretación, escucha) y la ingeniería cultural, en este caso la concepción de dispositivos hipermedia de asistencia a la escucha musical activa. Queda el devenir de lo que constituía el trasfondo de las conversaciones con A. Wisner y que voy a considerar aquí. Tras resumir someramente el tipo de antropología cognitiva desarrollada por el programa de investigación 'curso de acción' en relación con una ingeniería situacional y haberla resituado en un espacio de investigación más amplio en antropología cognitiva, pero que no abarca todo lo que denominamos "antropología cognitiva" (1), precisaré qué le debe esta antropología cognitiva a la antropología cultural y a la antropología de las técnicas, que no dudaré en agrupar para mi propósito bajo la expresión de "antropología cultural-técnica", pero también lo que les aporta o puede aportar por su parte (2). Concluiré con una retrospectiva y una prospectiva sobre las relaciones entre 
esta antropología cognitiva y la antropotecnología. En lo que respecta a los puntos (1) y (2) y teniendo en cuenta el espacio disponible, remitiré globalmente a las obras siguientes en las cuales se citan diversas publicaciones de investigaciones particulares o resúmenes parciales en varios ámbitos del programa de investigación 'curso de acción', así como diversas publicaciones de otras investigaciones sobre antropología cognitiva y cultural-técnica (Pinsky, 1992, Theureau et al., 1994, Theureau, 2003, 2004, 2006). En lo referente a la antropotecnología a la que Theureau (2006) se limita a hacer alusión, remitiré globalmente a las publicaciones de P. Geslin y a las tesis de doctorado sobre ergonomía que se dedican más especialmente a la antropotecnología dirigidas por A. Wisner en colaboración con otros, uno de los cuales yo mismo en una de ellas (Sagar, 1989).

\section{La enacción, la conciencia prerreflexiva, la actividad-signo, la ingeniería situacional y su contribución en la creación de un espacio de investigación en antropología cognitiva}

3 El programa de investigación 'curso de acción' aborda la actividad humana en sus situaciones naturales, por tanto culturales, de ejercicio como a la vez cognitiva, autónoma, encarnada, situada, al mismo tiempo individual y colectiva, técnicamente constituida, cultivada y vivida, tomando todas estas características hipotéticas en el más puro sentido:

- (1) cognitiva: es necesario una noción de saber para explicar la misma en términos a la vez de manifestación de saber y de constitución de saber;

- (2) autonónoma (u operacionalmente cerrada): consiste en una dinámica de acoplamiento estructural, es decir, en interacciones asimétricas entre un actor y su entorno (otros actores incluidos), es decir, en interacciones entre el actor considerado con quien, en este entorno, se ha seleccionado como pertinente para la organización interna en cada instante de este actor (mundo propio), interacciones cuyo propio contenido es pertinente para esta misma organización interna en cada instante (cuerpo propio);

- (3) encarnada: se rechaza cualquier separación entre cuerpo y mente. Así, según la expresión de S. Rose, neurofisiólogo, el término "mind" (mente) de hecho debe considerarse como un verbo, como "minding", y designar "lo que hacen el cuerpo y el cerebro";

- (4) situada dinámicamente en un mundo en el que existen otros actores: este mundo y estos otros actores contribuyen a esta actividad en la medida en que estos son pertinentes para la organización interna del actor considerado (véase más arriba).

- (5) a la vez individual y colectiva: esta participación de otros actores en la situación dinámica de un actor hace que la actividad individual de hecho sea individual-social, o incluso individual-colectiva, y que la actividad colectiva es de hecho social-individual, o incluso constantemente colectivizada y descolectivizada;

- (6) técnicamente constituida: dado que el mundo compartido por el actor considerado y por otros actores está técnicamente constituido, lo mismo ocurre con la actividad de este actor;

- (7) cultivada: la actividad está situada culturalmente, es decir, no separable de una cultura. La antropología cultural demuestra de hecho que si el conjunto de la humanidad comparte muchas cosas, la diferencia cultural afecta íntimamente a cada individuo; 
- (8) vivida: se necesita una noción de conciencia para explicar la actividad humana, la de conciencia prerreflexiva: la posibilidad, para el actor, de designar, imitar, contar y comentar su actividad a un cierto nivel mediante la reunión de condiciones favorables, acompaña en todo momento esta misma actividad y constituye una propiedad emergente del acoplamiento estructural.

4 Ante todo, lo que diferencia al programa de investigación 'curso de acción' de otros programas de investigación sobre antropología cognitiva que constituyen con éste un espacio de investigación sobre la actividad humana en sus situaciones naturales, por tanto culturales, de ejercicio, son las características (2) y (8), que condicionan la posibilidad de construir datos válidos sobre la actividad humana. De hecho, la característica (2), que constituye la esencia del paradigma cognitivo de la enacción, implica que un observador externo es incapaz de construir estos datos sobre lo que es pertinente para la organización interna en un instante dado del actor considerado, mientras que a la inversa, la característica (8) abre la posibilidad para este observador externo - más bien tendríamos que hablar de observador-interlocutor - de situar al actor en condiciones de construir tales datos. De ahí un observador que combina, según las posibilidades ofrecidas por las situaciones estudiadas y según la franja temporal del estudio, la observación y el registro del comportamiento y la recogida de verbalizaciones de los actores provocadas por los observadores-interlocutores (simultáneas, ligeramente diferidas, interruptivas, en autoconfrontación, en resituación por las huellas), a través del establecimiento previo y constantemente renovado, de condiciones materiales y temporales, éticas y sociopolíticas de cooperación de los actores en el estudio de su actividad. A excepción de los puntos (2) y (8), estos distintos puntos son compartidos por otros programas de investigación sobre antropología cognitiva que en la actualidad se pueden agrupar en cinco polos internacionales: (a) la acción (cognición) situada (que se ha impuesto con L. Suchman), (b) la cognición socialmente distribuida (desarrollada por E. Hutchins), (c) la teoría de la actividad (inspirada de lejos por L. Vygotsky), (d) la etnometodología actual (inspirada en un principio por H. Garfinkel, que apuntaba a una renovación metodológica de la sociología, fuera de cualquier preocupación cognitiva y técnica, pero que los trabajos de A. Cicourel, L. Suchman y otros han orientado hacia tales preocupaciones) y (e) la simple reorientación hacia el estudio cognitivo de las nociones y métodos de la antropología cultural-técnica (mientras que los demás polos, así como el programa de investigación 'curso de acción' los retoman pero asociándolos a otros). A estos cinco polos añadiré investigaciones (f) surgidas, como las investigaciones sobre los 'cursos de acción', de la ergonomía francesa, en particular las realizadas sobre las actividades cooperativas (iniciadas por B. Pavard). No obstante, algunas de estas características, las características (1) y (5) son compartidas en distintos grados por algunos de estos polos. Los polos (a), (b) y (d) sólo consideran el aspecto colectivo de la actividad humana, el único pertinente y analizable según las hipótesis que los controlan, no consideran, por tanto, más que una parte de la característica (5). El polo (c) puede inspirarse en L. Vygotsky para quien la actividad individual era individualsocial - de ahí, por otra parte, la formulación de la característica (5) -, pero en lo que respecta a la relación entre esta actividad individual-social y la actividad socialindividual, éste se limita a yuxtaponer análisis separados. Los polos (a), (b) y (d) sólo consideran, por tanto, el aspecto colectivo de la cognición humana, es decir una parte solamente de la característica (1). 
5 Al paso de estas diferencias, el programa de investigación 'curso de acción' desarrolla una fenomenología empírica de la actividad humana como "actividad-signo", a través de una noción de "signo hexádico" como descripción de la conciencia prerreflexiva asociada a una unidad de actividad significativa para el actor en un instante determinado, por tanto, también como descripción del conjunto de su actividad siempre que la misma dé lugar a conciencia prerreflexiva, que especifica y desarrolla la noción más amplia de in-formación (información formada desde el interior del actor humano, incluso de cualquier sistema vivo). Esta noción capta un conjunto de hipótesis empíricas generales de las cuales sólo señalaré aquí la principal: la actividad humana en cada instante produce, no una transformación de las representaciones del actor, sino una transformación de su estructura de anticipaciones. Esta fenomenología empírica de la actividad humana constituye un componente necesario de un método científico empírico que trate sobre esta actividad humana que parte de esta fenomenología para buscar sus limitaciones y efectos a la vez en los Cuerpos, las Situaciones (la cultura como materialidad compartida colectivamente en distintos grados en cada instante) y las Culturas (la cultura como hábito, incluso simbólicos, que emergen en la situación, ellos también compartidos colectivamente en distintos grados en cada instante) de los actores, y no sólo en uno u otro de estos tres ámbitos. Según este programa de investigación, esta fenomenología permite describir los fenómenos de la actividad humana de modo que se puedan formular explicaciones y evaluaciones válidas mientras que el conjunto de este método científico empírico permite hacer efectiva esta formulación. Esta fenomenología y este método científico producen modelos analíticos y sintéticos más o menos formalizados que tratan, a través de una cascada de objetos teóricos individuales-sociales y socio-individuales (en términos de articulaciones colectivas de actividades individuales-sociales), sobre la actividad individual-social y sobre la relación entre actividad individual-social y actividad social-individual. Estas se asocian a un programa de investigación tecnológica sobre ingeniería situacional, que difiere de la ingeniería usual, limitada a los artefactos, en que ésta se ocupa de la concepción de las situaciones, de un entorno dinámico incluyendo en su definición la actividad que se ejerce.

6 Esta fenomenología, este método científico y este método tecnológico han permitido, en relación con la extensión de los ámbitos empíricos y socio-técnicos, un desarrollo de las cuestiones empíricas y tecnológicas de las cuestiones de actividad individualsocial en producción nominal hacia las de aprendizaje-desarrollo y de apropriación-individuación de dispositivos técnicos, las de articulación entre actividad individual $\mathbf{y}$ actividad colectiva, las que tratan sobre las relaciones entre emoción, cognición y acción y, por último, sobre los procesos de creación, e incluso de salir de la investigación empírica y tecnológica para formular los principios de una epistemología normativa interna general asociando investigación empírica, investigación tecnológica, investigación matemática e investigación filosófica, sobre el fondo del "mundo de la vida" de los actores que llevan a cabo dichas investigaciones.

7 Este no es el lugar de explicitar más antes de este último párrafo, ni tampoco de discutir las ventajas e inconvenientes comparados de todas estas investigaciones sobre antropología cognitiva. Sin precisar más las hipótesis empíricas, los observatorios, las fenomenologías, los modelos analíticos y sintéticos y las aportaciones al concepto técnico-organizacional-cultural de estas investigaciones sobre antropología cognitiva, 
concluiré esta sección apuntando, cuando menos de un modo general, lo que, en estas investigaciones, condiciona las relaciones con la antropología cultural-técnica:

- o compartir una filiación común, la antropología cultural-técnica, combinada con otras filiaciones, la psicología - y, en particular, la psicología cognitiva experimental que ha impuesto el tema de la cognición en relación con la explotación de las posibilidades de la modelización informática y con la contribución a la concepción de herramientas y de entornos informáticos -, la sociología y la ergonomía;

aplicación de una vista dinámica (y no estática como en algunos otros trabajos de antropología cognitiva y en numerosos trabajos de antropología cultural realizados en términos de grupos culturales aislados) de la cultura a través de la consideración de la actividad humana;

- exploración sistemática de la cultura material y técnica en relación con la cultura en su conjunto;

- introducción de métodos de construcción de datos más intrusivos y basados en hipótesis más precisas que los métodos usuales de la antropología cultural-técnica;

introducción de situaciones de experimentación de campo y en situación simulada, diferentes de los experimentos de laboratorio (contrariamente a otros trabajos de antropología cognitiva);

- formulación de teorías más literales que van hasta modelos formales;

establecimiento de una relación con la concepción técnico-organizacional-cultural.

\section{Lo que esta antropología cognitiva debe a la antropología cultural y a la antropología de las técnicas y puede aportarles recíprocamente}

8 En general, esta antropología cognitiva se acerca más a los criterios de cientificidad de las ciencias físicas y biológicas que la antropología cultural-técnica de la cual procede en parte: literalización de las teorías (es decir, una formalización que permite engendrar hipótesis empíricas nuevas), proposiciones empíricas a vez no triviales y refutables por datos empíricos (por la utilización de métodos de construcción más formales de datos empíricos), relación orgánica con la técnica (incluyendo las herramientas y procesos de formación, de organización, de gestión y de logística y no sólo los artefactos). No obstante, su relación con la antropología cultural-técnica no se detiene ahí. De hecho, esta se debe a dos elementos esenciales de esta última.

9 En primer lugar, el estudio antropológico cultural-técnico constituye a la vez un paso previo y un horizonte del estudio antropológico cognitivo. De hecho, este constituye, por así decirlo, un "zoom hacia atrás" en el espacio socio-cultural y en el tiempo. En el espacio socio-cultural, por el hecho de que los estudios sobre antropología cognitiva sólo pueden concernir a un número relativamente restringido de actores en un lugar relativamente restringido (por ejemplo, en las investigaciones que he llevado a cabo sobre el manejo de reactores nucleares, el operador del reactor, el operador de agua-vapor y el supervisor, a los demás actores sólo se les consideraba en sus relaciones con los primeros). En el tiempo, por el hecho de que los estudios sobre antropología cognitiva sólo pueden considerar franjas temporales relativamente restringidas (por ejemplo, la franja temporal más amplia que he considerado en mis investigaciones ha sido de tres años de actividad de composición musical de un mismo compositor de música contemporánea, el resto de actores que intervenían en esta composición 
musical sólo se consideraban igualmente por sus relaciones con este compositor). El problema es primero que el mero inicio de estas investigaciones sobre antropología cognitiva requiere dicho "zoom hacia atrás" que, por sí mismo, puede permitir fundamentar científicamente lo que en ergonomía francesa se denomina el análisis de la demanda, adoptado por la antropotecnología. A este problema previo se añade un problema posterior que es que la explicación corporal, situacional y cultural de los fenómenos descritos con pertinencia y precisión a través de tales investigaciones sobre antropología cognitiva -por tanto también las conclusiones que una tal explicación permite deducir para la concepción técnico-organizacional-cultural - requiere dicho "zoom hacia atrás".

10 Seguidamente, el observatorio de las actividades humanas de la antropología cognitiva (sus métodos de construcción de datos y las hipótesis empíricas que los fundamentan) hereda de los debates epistemológicos de la antropología cultural-técnica. Como en ésta última, el observador-interlocutor de los actores en las investigaciones sobre antropología cognitiva constituye, por así decirlo, el "instrumento" esencial de construcción de datos. Son esenciales su epistemología normativa interna, y por tanto las expectativas ontológicas (relativas a la naturaleza de las cosas estudiadas) y éticopolíticas de la misma. De ahí la presencia, en el observatorio del programa de investigación 'curso de acción', de numerosos principios y elementos procedentes de la antropología cultural-técnica.

11 Recíprocamente, la antropología cognitiva aporta, o al menos puede aportar, varias contribuciones a la antropología cultural-técnica. Las primeras son obvias: dado que se interesa esencialmente en situaciones de los países occidentales, puede contribuir a traspasar los límites del exotismo; puede constituir un componente porque ésta última es a la vez su paso previo y su horizonte; por construcción, puede reforzar y hacer más detallada la contribución de la antropología cultural-técnica a la concepción técnicoorganizacional-cultural. Otra contribución menos evidente se basa en el hecho de que la rica teoría de la actividad individual y colectiva que es necesaria para la antropología cultural-técnica - como por otra parte, para la historia - pasa, al menos en parte, por los estudios empíricos precisos de la actividad humana realizados en antropología cognitiva.

\section{Conclusión}

12 Si se considera, para concluir, la antropotecnología, se puede constatar en primer lugar una comunidad de origen de esta última con al menos una parte de esta antropología cognitiva, el programa de investigación 'curso de acción' y las investigaciones sobre actividades cooperativas citadas en la sección 1: la antropología cultural-técnica y la ergonomía. Más ampliamente, la antropotecnología comparte con el conjunto de investigaciones sobre antropología cognitiva consideradas aquí una visión dinámica de la cultura y del vínculo entre cultura material, simbólica y comportamental, así como un interés por su transformación. Este hecho de compartir un mismo espacio de investigación permite cuando menos el desarrollo de debates teóricos y metodológicos fructíferos, así como la realización de investigaciones interdisciplinarias, e incluso complementarias (es decir, tras las cuales cada disciplina (o programa de investigación) sale no sólo transformado/a) sino también habiendo integrado algo del otro). 


\section{BIBLIOGRAFÍA}

Ombredane, A., \& Faverge, J.M. (1955). L'analyse du travail. Paris : PUF.

Pinsky, L. (1990). Définir l'ergonomie comme une technologie. In Actes du XXVIème Congrès Société d'Ergonomie de Langue Française, Montréal, 62-65.

Pinsky, L. (1992). Concevoir pour l'action et la communication: essais d'ergonomie cognitive. Berne : Peter Lang.

Pinsky, L., \& Theureau, J. (1987). L'étude du Cours d'Action. Analyse du travail et conception ergonomique. Rapport n 88, Paris : CNAM. (Coll. Physiologie du travail et d'Ergonomie).

Pinsky, L., Theureau, J., \& Sagar, M. (1989). Ergonomie et Anthropologie cognitive de l'action et de la communication dans le travail, Communication. In Actes des Premières journées Pirttem- CNRS de Psychologie du Travail- Ergonomie- Neurophysiologie du Travail, Paris, France.

Sagar, M. (1989). La conduite des dispositifs automatisés fonctionnant en mode dégradé- Modèle théorique et méthodologie d'analyse. Thèse de Doctorat d'Ergonomie, CNAM, Paris.

Theureau, J. (1990). Analyse du travail et Anthropologie cognitive. In Actes du XXVIème Congrès Société d'Ergonomie de Langue Française, Montréal.

Theureau, J. (2003). Course-of-action analysis \& course-of-action centered design. In E. Hollnagel (ed.), Handbook of cognitive task design (pp. 55-81). New Jersey: Lawrence Erlbaum Ass. Mahwah.

Theureau, J. (2004). Le cours d'action: Méthode élémentaire. Toulouse : Octarès.

Theureau, J. (2006). Le cours d'action : Méthode développée. Toulouse : Octarès.

Theureau, J., \& Jeffroy, F. (s/c) (1994) Ergonomie des situations informatisées : la conception centrée sur le cours d'action. Toulouse : Octarès.

\section{RESÚMENES}

Este texto contextualiza el programa antropotecnológico en el seno de las corrientes de la antropología cognitiva y explicita más concretamente los principios de esta antropología que han dado lugar al programa de investigación "curso de acción" y a la ingeniería situacional que, como la antropotecnología, se ha separado progresivamente de la ergonomía francesa. Desde finales de los años 80 hasta la actualidad, Theureau demuestra como el programa wisneriano ha coincidido, encontrado y comparte aún las preocupaciones surgidas por un análisis de la actividad prevista desde los principios de la antropología cultural, de la antropología de las técnicas pero también y sobre todo de una antropología cognitiva norteamericana. En este sentido, muestra aquí los puntos de encuentro, y de debate, teóricos y metodológicos que suscita la antropotecnología desde una perspectiva fenomenológica y enactiva del análisis de la actividad.

Este texto contextualiza o programa antropotecnológico no seio das correntes da antropologia cognitiva e explicita mais concretamente os princípios desta antropologia que originaram o programa de investigação sobre o "curso de ação" e a engenharia situacional que, tal como a antropotecnologia, se distanciou progressivamente da ergonomia de língua francesa. Desde o final dos anos 80 até à atualidade, Jacques Theaureau demonstra como o programa wisneriano partilha as preocupações desencadeadas por uma análise da atividade próxima da antropologia cultural, da antropologia das técnicas, mas também, e sobretudo, da antropologia cognitiva 
norte-americana. Neste sentido, mostra os pontos de encontro, os debates teóricos e metodológicos que suscita a antropotecnologia a partir da análise da atividade numa perspetiva fenomenológica e da enação.

Ce texte contextualise le programme anthropotechnologique au sein des courants de l'anthropologie cognitive. Il explicite plus particulièrement les principes de cette anthropologie qui ont donné lieu au programme de recherche sur «le cours d'action» qui, comme l'anthropotechnologie s'est progressivement détachée de l'ergonomie de langue française. Jacques Theureau montre comment le programme wisnerien partage les préoccupations soulevées par une analyse de l'activité proche de l'anthropologie cognitive. Il en précise les points de rencontre, les débats théoriques et méthodologiques.

This paper contextualizes the anthropotechnological program within cognitive anthropology, and particularly explicit principles of anthropology that gave rise to the research program on "course of action" which, as anthropotechnology gradually detached from the French speaking ergonomics. Since the late ' 80 s until now, Jacques Theureau shows how the wisnerien program fits, met and still shares the concerns raised by an «activity analysis » deeply influenced by cognitive anthropology.

\section{ÍNDICE}

Palabras claves: antropología cognitiva, antropotecnología, análisis de la actividad, curso de acción

Keywords: cognitive anthropology, enaction, anthropotechnology, activity analysis, « Cours d'action »

Palavras-chave: antropologia cognitiva, enação, antropotecnologia, análise da actividade, curso de ação

Mots-clés: anthropologie cognitive, enaction, anthropotechnologie, analyse de l'activité, cours d'action

\section{AUTOR}

\section{JACQUES THEUREAU}

Equipo de investigación "Análisis de las Prácticas Musicales" UMR 9912 STMS (Ciencias y Técnicas de la Música y del Sonido) , IRCAM-CNRS (Institut de Recherche et Coordination Acoustique/ Musique - Centre National de la Recherche Scientifique), 1, place Igor-Stravinsky, 75004 Paris theureau@ircam.fr 